\title{
New inertial relaxed method for solving split feasibilities
}

\author{
Yekini Shehu ${ }^{1,2}$ (D) Aviv Gibali ${ }^{3,4}$
}

Received: 18 January 2020 / Accepted: 21 May 2020 / Published online: 2 June 2020

(c) The Author(s) 2020

\section{Abstract}

In this paper, we introduce a relaxed CQ method with alternated inertial step for solving split feasibility problems. We give convergence of the sequence generated by our method under some suitable assumptions. Some numerical implementations from sparse signal and image deblurring are reported to show the efficiency of our method.

Keywords Alternated inertial · CQ methods · Split feasibility problems · Signal processing

\section{Introduction}

Censor and Elfving [12] introduced the following Split Convex Feasibility Problem (SCFP), see also [11],

$$
\text { find } x \in C \text { such that } A x \in Q \text {, }
$$

where $A: \mathbb{R}^{k} \rightarrow \mathbb{R}^{m}$ is a bounded and linear operator, $C \subseteq \mathbb{R}^{k}$ and $Q \subseteq \mathbb{R}^{m}$ are nonempty, closed and convex sets. Hereafter, we let $S$ represent the set of solutions to SCFP (1).

Originally the SCFP was introduced in Euclidean spaces, and afterwards extended to infinite dimensional spaces as well as applied successfully in the field of intensitymodulated radiation therapy (IMRT) treatment planning, see [11-13,15].

\footnotetext{
$\bowtie$ Yekini Shehu

yekini.shehu@unn.edu.ng

Aviv Gibali

avivg@braude.ac.il

1 Department of Mathematics, Zhejiang Normal University, Jinhua 321004, China

2 Institute of Science and Technology (IST), Am Campus 1, 3400 Klosterneuburg, Austria

3 Department of Mathematics, ORT Braude College, 2161002 Karmiel, Israel

4 The Center for Mathematics and Scientific Computation, University of Haifa, 3498838 Mt. Carmel, Haifa, Israel
} 
Since the introduction of the SCFP, many authors have introduced various iterative methods for solving it, see, for example, [10,14,17-19,23,29,30,38,41-43,49,52]. It is known that (see, e.g., [9]) $x \in C$ solves the SCFP (1) if and only if $x$ solves the fixed point problem:

$$
x=P_{C}\left(x-\lambda A^{t}\left(I-P_{Q}\right) A x\right), \lambda>0
$$

and consequently, the following Byrne's CQ method [9] was introduced:

$$
x_{n+1}=P_{C}\left(x_{n}-\lambda A^{t}\left(I-P_{Q}\right) A x_{n}\right), n \geq 1
$$

where $A^{t}$ denotes the transpose of $A$.

Weak convergence of the CQ method is guaranteed under the assumption that $\lambda \in$ $\left(0,2 /\|A\|^{2}\right)$. So, an implementation of (2) requires a norm estimation of the bounded linear operator $A$, or the spectral radius of the matrix $A^{t} A$ in finite-dimensional framework. This fact might effect the applicability of the method in practice, see [26, Theorem 2.3]. So, in order to circumvent this scenario, López et al. [30] introduced a modification of the CQ method (2) by replacing the step-size $\lambda$ in (2) with the following adaptive step:

$$
\tau_{n}=\frac{\rho_{n} f\left(x_{n}\right)}{\left\|\nabla f\left(x_{n}\right)\right\|^{2}}, \quad n \geq 1,
$$

where $\rho_{n} \in(0,4), f\left(x_{n}\right)=\frac{1}{2}\left\|\left(I-P_{Q}\right) A x_{n}\right\|^{2}$ and $\nabla f\left(x_{n}\right)=A^{t}\left(I-P_{Q}\right) A x_{n}$ for all $n \geq 1$. There exists many other modifications of the CQ algorithm, see, for example, [20,23,24,44,49].

Following the heavy ball method of Polyak [39], Nesterov [37] introduced the following iterative step:

$$
\begin{aligned}
y_{n} & =x_{n}+\theta_{n}\left(x_{n}-x_{n-1}\right), \\
x_{n+1} & =y_{n}-\lambda_{n} \nabla f\left(y_{n}\right), \quad n \geq 1,
\end{aligned}
$$

where $\theta_{n} \in[0,1)$ is an inertial factor and $\lambda_{n}$ is a positive sequence. It was shown via numerical experiments in the field of image reconstruction, that (4) and other associated methods, such as [1,2,6-8,18,21,31,32,34], have greatly improved the performance of their non-inertial algorithms, that is, when $\theta_{n}=0$. Hence this idea is also referred to as inertial algorithms.

In this spirit, several inertial-type methods for solving SCFPs have been proposed recently, see [16,44-47,51], just to name a few. In particular, Dang et al. [18] (see also [17]) proposed the following inertial relaxed CQ algorithms for solving SCFPs:

$$
x_{n+1}=P_{C_{n}}\left(I-\lambda A^{t}\left(I-P_{Q_{n}}\right) A y_{n}\right) \text {, }
$$

and

$$
x_{n+1}=\left(1-\alpha_{n}\right) y_{n}+\alpha_{n} P_{C_{n}}\left(I-\lambda A^{t}\left(I-P_{Q_{n}}\right) A y_{n}\right)
$$


where $y_{n}=x_{n}+\theta_{n}\left(x_{n}-x_{n-1}\right), \alpha_{n} \in(0,1), \lambda \in\left(0,2 /\|A\|^{2}\right)$ and $\theta_{n} \in\left[0, \bar{\theta}_{n}\right]$ with $\bar{\theta}_{n}:=\min \left\{\theta,\left(\max \left\{n^{2}\left\|x_{n}-x_{n-1}\right\|^{2}, n^{2}\left\|x_{n}-x_{n-1}\right\|\right\}\right)^{-1}\right\}, \theta \in[0,1)$.

An important observation regarding the above inertial methods [16-18,44-47,51], is that the sequence $\left\{x_{n}\right\}$ generated by these inertial-type methods does not have a monotonic behaviour with respect to $x^{*} \in S$ and can move or swing back and forth around $S$, see, for example, [5,31]. This could explain why such inertial extrapolation step does not converge faster than its counterpart non-inertial methods, see, e.g., [33]. In a direction to resolve the above issue, an alternated inertial method was introduced recently in [36]. This alternated inertia method shown to exhibit attractive performances in practice including monotonicity of $\left\{\left\|x_{2 n}-x^{*}\right\|\right\}$, see [27,28] for more details.

Motivated by the above works, we propose a new relaxed CQ method with alternated inertial procedure for solving SCFPs. We establish global convergence of our scheme under some easy to verify assumptions. Moreover, the parameters controlling the inertial factor, that is, $\theta_{n}$ can be chosen as close as possible to 1 (when $\mu$ tends to zero in (10)). This is opposite to many other related methods that restrict it to less than 1 , see, e.g., [16-18,44-47,51].

The outline of the paper is a follows. Definitions, basic concepts and useful results are presented in Sect. 2. The method and its analysis is given in Sect. 3 and then some numerical experiments in the field of signal processing which illustrate the effectiveness and applicability of our proposed scheme is presented in Sect. 4. Final remarks are presented in Sect. 5.

\section{Preliminaries}

We start by recalling some definitions and basic results.

A mapping $T: \mathbb{R}^{k} \rightarrow \mathbb{R}^{k}$ is called

(a) nonexpansive if $\|T x-T y\| \leq\|x-y\|$, for all $x, y \in \mathbb{R}^{k}$;

(b) firmly nonexpansive if $\|T x-T y\|^{2} \leq\|x-y\|^{2}-\|(I-T) x-(I-T) y\|^{2}$ for all $x, y \in \mathbb{R}^{k}$. Equivalently, $\|T x-T y\|^{2} \leq\langle x-y, T x-T y\rangle$ for all $x, y \in \mathbb{R}^{k}$.

It is shown in [25] that $T$ is firmly nonexpansive if and only if $I-T$ is firmly nonexpansive.

Let $C$ be a nonempty, closed and convex subset of $\mathbb{R}^{k}$. For any point $u \in \mathbb{R}^{k}$, there exists a unique point $P_{C} u \in C$ such that

$$
\left\|u-P_{C} u\right\| \leq\|u-y\| \forall y \in C,
$$

$P_{C}$ is called the metric projection of $\mathbb{R}^{k}$ onto $C$. Some important properties of the metric projection are listed next, for this and more see [4]. We know that $P_{C}$ is a firmly nonexpansive mapping of $\mathbb{R}^{k}$ onto $C$. It is also known that $P_{C}$ satisfies

$$
\left\langle x-y, P_{C} x-P_{C} y\right\rangle \geq\left\|P_{C} x-P_{C} y\right\|^{2} \forall x, y \in \mathbb{R}^{k} .
$$


Furthermore, $P_{C} x$ is characterized by the property

$$
P_{C} x \in C \text { and }\left\langle x-P_{C} x, P_{C} x-y\right\rangle \geq 0 \forall y \in C .
$$

This characterization implies that

$$
\|x-y\|^{2} \geq\left\|x-P_{C} x\right\|^{2}+\left\|y-P_{C} x\right\|^{2} \forall x \in \mathbb{R}^{k}, \forall y \in C .
$$

Let the function $f: \mathbb{R}^{k} \rightarrow \mathbb{R}$, an element $g \in \mathbb{R}^{k}$ is said to be a subgradient of $f$ at $x$ if

$$
f(y) \geq f(x)+\langle y-x, g\rangle, \quad \forall y \in \mathbb{R}^{k}
$$

The subdifferential of $f$ at $x, \partial f(x)$, is defined by

$$
\partial f(x):=\left\{g \in \mathbb{R}^{k}: f(y) \geq f(x)+\langle y-x, g\rangle, \quad \forall y \in \mathbb{R}^{k}\right\} .
$$

Lemma 2.1 ([10]) Let $C$ be nonempty, closed and convex subset of $\mathbb{R}^{k}$ and $x \in \mathbb{R}^{k}$. Consider the function $f(x):=\frac{1}{2}\left\|\left(I-P_{Q}\right) A x\right\|^{2}$, then

(i) the function $f$ is convex and differentiable.

(ii) The gradient of $f$ at $x$ is defined as $\nabla f(x)=A^{t}\left(I-P_{Q}\right) A x$.

(iii) $\nabla f$ is $\|A\|^{2}$-Lipschitz continuous.

The next basic lemma is useful for our analysis.

Lemma 2.2 Let $x, y \in \mathbb{R}^{k}$. Then

(i) $\|x+y\|^{2}=\|x\|^{2}+2\langle x, y\rangle+\|y\|^{2}$;

(ii) $\|x+y\|^{2} \leq\|x\|^{2}+2\langle y, x+y\rangle$;

(iii) $\|\alpha x+\beta y\|^{2}=\alpha(\alpha+\beta)\|x\|^{2}+\beta(\alpha+\beta)\|y\|^{2}-\alpha \beta\|x-y\|^{2}, \quad \forall \alpha, \beta \in \mathbb{R}$.

\section{The algorithm}

In the light of [22], we consider a relaxed CQ method with alternated inertial extrapolation step in which $C$ and $Q$ in (1) are level sets of convex functions given by

$$
C:=\left\{x \in \mathbb{R}^{k}: c(x) \leq 0\right\}
$$

and

$$
Q:=\left\{x \in \mathbb{R}^{m}: q(x) \leq 0\right\}
$$

where $c: \mathbb{R}^{k} \rightarrow \mathbb{R}$ and $q: \mathbb{R}^{m} \rightarrow \mathbb{R}$ are convex functions. By [3, Fact 7.2 (iii)], $c$ and $q$ are subdifferentiable on $C$ and $Q$, respectively, and $c$ and $q$ are bounded on bounded sets. 
For $n \geq 1$, define

$$
C_{n}:=\left\{x \in \mathbb{R}^{k}: c\left(w_{n}\right) \leq\left\langle\xi_{n}, w_{n}-x\right\rangle\right\}
$$

and

$$
Q_{n}:=\left\{y \in \mathbb{R}^{m}: q\left(A w_{n}\right) \leq\left\langle\zeta_{n}, A w_{n}-y\right\rangle\right\}
$$

with $\xi_{n} \in \partial c\left(w_{n}\right)$ and $\zeta_{n} \in \partial q\left(A w_{n}\right)$, respectively. It can be easily seen that $C_{n} \supset C$ and $Q_{n} \supset Q$ for all $n$. Consequently, since $C_{n}$ and $Q_{n}$ are two half-spaces, the projections onto these sets have a closed formulas and hence easy to compute. From now on we define for all $x \in \mathbb{R}^{k}: f_{n}(x):=\frac{1}{2}\left\|\left(I-P_{Q_{n}}\right) A x\right\|^{2}$ and $\nabla f_{n}(x)=$ $A^{t}\left(I-P_{Q_{n}}\right) A x$.

\section{Algorithm 1 Relaxed CQ Method with inertial extrapolation step}

1: Given $\gamma>0, l \in(0,1), \mu \in(0,1)$, choose the parameter $\theta_{n}$ such that

$$
0 \leq \theta_{n} \leq \theta<\frac{1-\mu}{1+\mu} .
$$

Choose starting points $x_{0}, x_{1} \in \mathbb{R}^{k}$ and set $n:=1$.

2: Given the iterations $x_{n}, x_{n-1}$, compute

$$
w_{n}= \begin{cases}x_{n}, & n=\text { even } \\ x_{n}+\theta_{n}\left(x_{n}-x_{n-1}\right), & n=\text { odd } .\end{cases}
$$

3: Compute

$$
\bar{x}_{n}=P_{C_{n}}\left(w_{n}-\tau_{n} \nabla f_{n}\left(w_{n}\right)\right),
$$

where $\tau_{n}=\gamma l^{m_{n}}$ and $m_{n}$ is the smallest non-negative integer $m$ such that

$$
\tau_{n}\left\|\nabla f_{n}\left(w_{n}\right)-\nabla f_{n}\left(\bar{x}_{n}\right)\right\| \leq \mu\left\|w_{n}-\bar{x}_{n}\right\| .
$$

4: Calculate the next iterate via

$$
x_{n+1}=P_{C_{n}}\left(w_{n}-\tau_{n} \nabla f_{n}\left(\bar{x}_{n}\right)\right) \text {. }
$$

5: Set $n \leftarrow n+1$, and go to 2 .

Remark 3.1 (a) As mentioned in the introduction, by adding the inertial extrapolation step in (5) and (6) to the classical CQ algorithm (2), the new generated sequence $\left\{x_{n}\right\}$ can move or swing back and forth around $S$ and hence we do not have monotonicity of $\left\{\left\|x_{n}-x^{*}\right\|\right\}, x^{*} \in S$. This matter can affect the convergence speed of the CQ methods with inertial extrapolation step, and sometimes would not even converge faster than the original CQ methods. In order to circumvent this scenario and regain monotonicity to some extent (see Lemma 3.2 below), we introduce the inertial extrapolation step (11). 
(b) Observe that if $\theta_{n}=0$, then Algorithm 1 reduces to the methods proposed in $[23,40,50]$.

(c) Our scheme allows to choose the parameters controlling the inertial factor $\theta_{n}$ as close as possible to 1 , when $\mu$ tends to zero in (10). This is more flexible than the methods in [16-18,44-47,51]. In general a wise choice of $\theta_{n}$ in Step 2 of Algorithm 1 enables acceleration of our method.

(d) Observe that we make use of an Armijo line search rule Algorithm 1, which is similar to [23] and hence following [23, Lemma 3.1], the search rule in Algorithm 1 ends after a finite number of iterations. Furthermore,

$$
\frac{\mu l}{\|A\|^{2}}<\tau_{n} \leq \gamma, \forall n \geq 1
$$

\subsection{Convergence Analysis}

We give the convergence analysis of Algorithm 1 under the assumption that the solution set of the SCFP (1) is nonempty.

Lemma 3.2 Suppose that the solution set of the SCFP (1) is nonempty, that is, $S \neq \emptyset$ and $\left\{x_{n}\right\}$ is any sequence generated by Algorithm 1. Then $\left\{x_{2 n}\right\}$ is Fejér monotone with respect to $S$ (i.e., $\left\|x_{2 n+2}-z\right\| \leq\left\|x_{2 n}-z\right\|, \forall z \in S$ ).

Proof Pick a point $z$ in $S$. Then

$$
\begin{aligned}
&\left\|x_{2 n+2}-z\right\|^{2}=\left\|P_{C_{2 n+1}}\left(w_{2 n+1}-\tau_{2 n+1} \nabla f_{2 n+1}\left(\bar{x}_{2 n+1}\right)\right)-z\right\|^{2} \\
& \leq\left\|\left(w_{2 n+1}-z\right)-\tau_{2 n+1} \nabla f_{2 n+1}\left(\bar{x}_{2 n+1}\right)\right\|^{2}-\| x_{2 n+2}-w_{2 n+1} \\
&+\tau_{2 n+1} \nabla f_{2 n+1}\left(\bar{x}_{2 n+1}\right) \|^{2} \\
&=\left\|w_{2 n+1}-z\right\|^{2}-2 \tau_{2 n+1}\left\langle\nabla f_{2 n+1}\left(\bar{x}_{2 n+1}\right), w_{2 n+1}-z\right\rangle-\left\|x_{2 n+2}-w_{2 n+1}\right\|^{2} \\
&-2 \tau_{2 n+1}\left\langle\nabla f_{2 n+1}\left(\bar{x}_{2 n+1}\right), x_{2 n+2}-w_{2 n+1}\right\rangle \\
&=\left\|w_{2 n+1}-z\right\|^{2}-2 \tau_{2 n+1}\left\langle\nabla f_{2 n+1}\left(\bar{x}_{2 n+1}\right), w_{2 n+1}-\bar{x}_{2 n+1}\right\rangle \\
&-2 \tau_{2 n+1}\left\langle\nabla f_{2 n+1}\left(\bar{x}_{2 n+1}\right), \bar{x}_{2 n+1}-z\right\rangle-\left\|x_{2 n+2}-w_{2 n+1}\right\|^{2} \\
&-2 \tau_{2 n+1}\left\langle\nabla f_{2 n+1}\left(\bar{x}_{2 n+1}\right), x_{2 n+2}-w_{2 n+1}\right\rangle \\
&=\left\|w_{2 n+1}-z\right\|^{2}-2 \tau_{2 n+1}\left\langle\nabla f_{2 n+1}\left(\bar{x}_{2 n+1}\right), \bar{x}_{2 n+1}-z\right\rangle \\
&-2 \tau_{2 n+1}\left\langle\nabla f_{2 n+1}\left(\bar{x}_{2 n+1}\right), x_{2 n+2}-\bar{x}_{2 n+1}\right\rangle \\
&-\left\|x_{2 n+2}-\bar{x}_{2 n+1}+\bar{x}_{2 n+1}-w_{2 n+1}\right\|^{2} \\
&=\left\|w_{2 n+1}-z\right\|^{2}-2 \tau_{2 n+1}\left\langle\nabla f_{2 n+1}\left(\bar{x}_{2 n+1}\right), \bar{x}_{2 n+1}-z\right\rangle-\left\|x_{2 n+2}-\bar{x}_{2 n+1}\right\|^{2} \\
&-\left\|\bar{x}_{2 n+1}-w_{2 n+1}\right\|^{2}-2\left\langle\bar{x}_{2 n+1}-w_{2 n+1}\right. \\
&\left.+\tau_{2 n+1} \nabla f_{2 n+1}\left(\bar{x}_{2 n+1}\right), x_{2 n+2}-\bar{x}_{2 n+1}\right\rangle .
\end{aligned}
$$

By the fact that $I-P_{Q_{2 n+1}}$ is firmly-nonexpansive and $\nabla f_{2 n+1}(z)=0$, we get

$$
\begin{aligned}
& 2 \tau_{2 n+1}\left\langle\nabla f_{2 n+1}\left(\bar{x}_{2 n+1}\right), \bar{x}_{2 n+1}-z\right\rangle=2 \tau_{2 n+1}\left\langle\nabla f_{2 n+1}\left(\bar{x}_{2 n+1}\right)\right. \\
& \left.\quad-\nabla f_{2 n+1}(z), \bar{x}_{2 n+1}-z\right\rangle
\end{aligned}
$$




$$
\begin{aligned}
& =2 \tau_{2 n+1}\left\langle A^{t}\left(I-P_{Q_{2 n+1}}\right) A \bar{x}_{2 n+1}-A^{t}\left(I-P_{Q_{2 n+1}}\right) A z, \bar{x}_{2 n+1}-z\right\rangle \\
& =2 \tau_{2 n+1}\left\langle\left(I-P_{Q_{2 n+1}}\right) A \bar{x}_{2 n+1}-\left(I-P_{Q_{2 n+1}}\right) A z, A \bar{x}_{2 n+1}-A z\right\rangle \\
& \geq \frac{2 \mu l}{\|A\|^{2}}\left\|\left(I-P_{Q_{2 n+1}}\right) A \bar{x}_{2 n+1}\right\|^{2} .
\end{aligned}
$$

By (8) and the fact that $\bar{x}_{2 n+1} \in C_{2 n+1}$, we get

$$
\left\langle\bar{x}_{2 n+1}-w_{2 n+1}+\tau_{2 n+1} \nabla f_{2 n+1}\left(w_{2 n+1}\right), x_{2 n+2}-\bar{x}_{2 n+1}\right\rangle \geq 0 .
$$

Consequently,

$$
\begin{aligned}
- & \left\langle\bar{x}_{2 n+1}-w_{2 n+1}+\tau_{2 n+1} \nabla f_{2 n+1}\left(\bar{x}_{2 n+1}\right), x_{2 n+2}-\bar{x}_{2 n+1}\right\rangle \\
\leq & 2\left\langle w_{2 n+1}-\bar{x}_{2 n+1}-\tau_{2 n+1} \nabla f_{2 n+1}\left(\bar{x}_{2 n+1}\right), x_{2 n+2}-\bar{x}_{2 n+1}\right\rangle \\
& +2\left\langle\bar{x}_{2 n+1}-w_{2 n+1}+\tau_{2 n+1} \nabla f_{2 n+1}\left(w_{2 n+1}\right), x_{2 n+2}-\bar{x}_{2 n+1}\right\rangle \\
= & 2 \tau_{2 n+1}\left\langle\nabla f_{2 n+1}\left(w_{2 n+1}\right)-\nabla f_{2 n+1}\left(\bar{x}_{2 n+1}\right), x_{2 n+2}-\bar{x}_{2 n+1}\right\rangle \\
\leq & 2 \tau_{2 n+1}\left\|\nabla f_{2 n+1}\left(w_{2 n+1}\right)-\nabla f_{2 n+1}\left(\bar{x}_{2 n+1}\right)\right\|\left\|x_{2 n+2}-\bar{x}_{2 n+1}\right\| \\
\leq & \tau_{2 n+1}^{2}\left\|\nabla f_{2 n+1}\left(w_{2 n+1}\right)-\nabla f_{2 n+1}\left(\bar{x}_{2 n+1}\right)\right\|^{2}+\left\|x_{2 n+2}-\bar{x}_{2 n+1}\right\|^{2} \\
\leq & \mu^{2}\left\|w_{2 n+1}-\bar{x}_{2 n+1}\right\|^{2}+\left\|x_{2 n+2}-\bar{x}_{2 n+1}\right\|^{2}
\end{aligned}
$$

Using (15), (16) and (17) in (14):

$$
\begin{aligned}
\left\|x_{2 n+2}-z\right\|^{2} \leq & \left\|w_{2 n+1}-z\right\|^{2}-\frac{2 \mu l}{\|A\|^{2}}\left\|\left(I-P_{Q_{2 n+1}}\right) A \bar{x}_{2 n+1}\right\|^{2} \\
& -\left(1-\mu^{2}\right)\left\|w_{2 n+1}-\bar{x}_{2 n+1}\right\|^{2} .
\end{aligned}
$$

Now,

$$
\begin{aligned}
\left\|w_{2 n+1}-z\right\|^{2}= & \left\|x_{2 n+1}+\theta_{2 n+1}\left(x_{2 n+1}-x_{2 n}\right)-z\right\|^{2} \\
= & \left\|\left(1+\theta_{2 n+1}\right)\left(x_{2 n+1}-z\right)-\theta_{2 n+1}\left(x_{2 n}-z\right)\right\|^{2} \\
= & \left(1+\theta_{2 n+1}\right)\left\|x_{2 n+1}-z\right\|^{2}-\theta_{2 n+1}\left\|x_{2 n}-z\right\|^{2} \\
& +\theta_{2 n+1}\left(1+\theta_{2 n+1}\right)\left\|x_{2 n+1}-x_{2 n}\right\|^{2} .
\end{aligned}
$$

Using similar arguments in showing (18), one can get

$$
\begin{aligned}
\left\|x_{2 n+1}-z\right\|^{2} \leq & \left\|w_{2 n}-z\right\|^{2}-\frac{2 \mu l}{\|A\|^{2}}\left\|\left(I-P_{Q_{2 n}}\right) A \bar{x}_{2 n}\right\|^{2} \\
& -\left(1-\mu^{2}\right)\left\|w_{2 n}-\bar{x}_{2 n}\right\|^{2} \\
= & \left\|x_{2 n}-z\right\|^{2}-\frac{2 \mu l}{\|A\|^{2}}\left\|\left(I-P_{Q_{2 n}}\right) A \bar{x}_{2 n}\right\|^{2} \\
& -\left(1-\mu^{2}\right)\left\|w_{2 n}-\bar{x}_{2 n}\right\|^{2} .
\end{aligned}
$$


Putting (20) and (19) into (18):

$$
\begin{aligned}
\left\|x_{2 n+2}-z\right\|^{2} \leq & \left\|x_{2 n}-z\right\|^{2}-\frac{2 \mu l}{\|A\|^{2}}\left(1+\theta_{2 n+1}\right)\left\|\left(I-P_{Q_{2 n}}\right) A \bar{x}_{2 n}\right\|^{2} \\
& -\left(1-\mu^{2}\right)\left(1+\theta_{2 n+1}\right)\left\|w_{2 n}-\bar{x}_{2 n}\right\|^{2} \\
& +\theta_{2 n+1}\left(1+\theta_{2 n+1}\right)\left\|x_{2 n+1}-x_{2 n}\right\|^{2} \\
& -\frac{2 \mu l}{\|A\|^{2}}\left\|\left(I-P_{Q_{2 n+1}}\right) A \bar{x}_{2 n+1}\right\|^{2} \\
& -\left(1-\mu^{2}\right)\left\|w_{2 n+1}-\bar{x}_{2 n+1}\right\|^{2}
\end{aligned}
$$

Observe that

$$
\begin{aligned}
\left\|x_{2 n+1}-x_{2 n}\right\| & \leq\left\|x_{2 n+1}-\bar{x}_{2 n}\right\|+\left\|x_{2 n}-\bar{x}_{2 n}\right\| \\
& \leq\left\|w_{2 n}-\tau_{2 n} \nabla f_{2 n}\left(\bar{x}_{2 n}\right)-w_{2 n}+\tau_{2 n} \nabla f_{2 n}\left(w_{2 n}\right)\right\|+\left\|x_{2 n}-\bar{x}_{2 n}\right\| \\
& \leq \tau_{2 n}\left\|\nabla f_{2 n}\left(\bar{x}_{2 n}\right)-\nabla f_{2 n}\left(w_{2 n}\right)\right\|+\left\|x_{2 n}-\bar{x}_{2 n}\right\| \\
& \leq(1+\mu)\left\|x_{2 n}-\bar{x}_{2 n}\right\| .
\end{aligned}
$$

Using (22) in (21):

$$
\begin{aligned}
\left\|x_{2 n+2}-z\right\|^{2} \leq & \left\|x_{2 n}-z\right\|^{2}-\frac{2 \mu l}{\|A\|^{2}}\left(1+\theta_{2 n+1}\right)\left\|\left(I-P_{Q_{2 n}}\right) A \bar{x}_{2 n}\right\|^{2} \\
& -\left[\left(1-\mu^{2}\right)\left(1+\theta_{2 n+1}\right)-\theta_{2 n+1}\left(1+\theta_{2 n+1}\right)(1+\mu)^{2}\right]\left\|w_{2 n}-\bar{x}_{2 n}\right\|^{2} \\
& -\frac{2 \mu l}{\|A\|^{2}}\left\|\left(I-P_{Q_{2 n+1}}\right) A \bar{x}_{2 n+1}\right\|^{2}-\left(1-\mu^{2}\right)\left\|w_{2 n+1}-\bar{x}_{2 n+1}\right\|^{2} \\
\leq & \left\|x_{2 n}-z\right\|^{2} .
\end{aligned}
$$

Therefore,

$$
\left\|x_{2 n+2}-z\right\| \leq\left\|x_{2 n}-z\right\| .
$$

Theorem 3.3 Suppose that $S \neq \varnothing$ and $\left\{x_{n}\right\}$ is any sequence generated by Algorithm 1. Then $\left\{x_{n}\right\}$ converges to a point in $S$.

Proof By Lemma 3.2, we have that $\lim _{n \rightarrow \infty}\left\|x_{2 n}-z\right\|$ exists and this implies that $\left\{x_{2 n}\right\}$ is bounded. Furthermore, we get from (23) that

$$
\lim _{n \rightarrow \infty}\left\|\left(I-P_{Q_{2 n}}\right) A \bar{x}_{2 n}\right\|=0 .
$$

From (23)

$$
\lim _{n \rightarrow \infty}\left\|x_{2 n}-\bar{x}_{2 n}\right\|=0
$$


Now, since $\left(I-P_{Q_{2 n}}\right)$ is nonexpansive,

$$
\begin{aligned}
\left\|\left(I-P_{Q_{2 n}}\right) A \bar{x}_{2 n}\right\| \leq & \left\|\left(I-P_{Q_{2 n}}\right) A x_{2 n}-\left(I-P_{Q_{2 n}}\right) A \bar{x}_{2 n}\right\| \\
& +\left\|\left(I-P_{Q_{2 n}}\right) A \bar{x}_{2 n}\right\| \\
\leq & \left\|A x_{2 n}-A \bar{x}_{2 n}\right\|+\left\|\left(I-P_{Q_{2 n}}\right) A \bar{x}_{2 n}\right\| \\
\leq & \|A\|\left\|x_{2 n}-\bar{x}_{2 n}\right\|+\left\|\left(I-P_{Q_{2 n}}\right) A \bar{x}_{2 n}\right\| .
\end{aligned}
$$

By (24) and (25), we get from (26) that

$$
\lim _{n \rightarrow \infty}\left\|\left(I-P_{Q_{2 n}}\right) A x_{2 n}\right\|=0 .
$$

Similarly, just like (27), one can show that

$$
\lim _{n \rightarrow \infty}\left\|\left(I-P_{Q_{2 n+1}}\right) A x_{2 n+1}\right\|=0 .
$$

Since $\partial q$ is bounded on bounded sets, we get $\delta>0$ such that $\left\|\zeta_{2 n}\right\| \leq \delta$. Since $P_{Q_{2 n}} A x_{2 n} \in Q_{2 n}$, we obtain from Algorithm 1 that

$$
\begin{aligned}
q\left(A x_{2 n}\right) & \leq\left\langle\zeta_{2 n}, A x_{2 n}-P_{Q_{2 n}} A x_{2 n}\right\rangle \\
& \leq \delta\left\|\left(I-P_{Q_{2 n}}\right) A x_{2 n}\right\| \rightarrow 0, n \rightarrow \infty .
\end{aligned}
$$

Since $\left\{x_{2 n}\right\}$ is bounded, there exists $\left\{x_{2 n_{j}}\right\} \subset\left\{x_{2 n}\right\}$ such that $x_{2 n_{j}} \rightarrow x^{*} \in \mathbb{R}^{k}$. Then continuity of $q$ and (29) imply

$$
q\left(x^{*}\right) \leq \liminf _{n \rightarrow \infty} q\left(A x_{2 n_{j}}\right) \leq 0 .
$$

Thus, $A x^{*} \in Q$.

By (22) and (23), we get

$$
\lim _{n \rightarrow \infty}\left\|x_{2 n+1}-x_{2 n}\right\|=0
$$

Since $x_{2 n_{j}+1} \in C_{2 n_{j}}$, then by definition of $C_{2 n_{j}}$,

$$
c\left(w_{2 n_{j}}\right)+\left\langle\xi_{2 n_{j}}, x_{2 n_{j}+1}-w_{2 n_{j}}\right\rangle \leq 0,
$$

where $\xi_{2 n_{j}} \in \partial c\left(x_{2 n_{j}}\right)$. By the boundedness of $\left\{\xi_{2 n_{j}}\right\}$ and (30), we get

$$
\begin{aligned}
c\left(x_{2 n_{j}}\right)=c\left(w_{2 n_{j}}\right) & \leq\left\langle\xi_{2 n_{j}}, w_{2 n_{j}}-x_{2 n_{j}+1}\right\rangle \\
& \leq\left\|\xi_{2 n_{j}}\right\|\left\|w_{2 n_{j}}-x_{2 n_{j}+1}\right\| \\
& =\left\|\xi_{2 n_{j}}\right\|\left\|x_{2 n_{j}}-x_{2 n_{j}+1}\right\| \rightarrow 0, j \rightarrow \infty .
\end{aligned}
$$

By continuity of $c$ and $x_{2 n_{j}} \rightarrow x^{*}$, we get from (31) that

$$
c\left(x^{*}\right) \leq \liminf _{j \rightarrow \infty} c\left(x_{2 n_{j}}\right) \leq 0 .
$$


Thus, $x^{*} \in C$. Therefore, $x^{*} \in S$.

We next show that the sequence of odd terms $\left\{x_{2 n+1}\right\}$ converges to $x^{*}$. Note that since $\lim _{n \rightarrow \infty}\left\|x_{2 n}-x^{*}\right\|$ exists and $\lim _{j \rightarrow \infty}\left\|x_{2 n_{j}}-x^{*}\right\|=0$, we get $\lim _{n \rightarrow \infty}\left\|x_{2 n}-x^{*}\right\|=0$.

Therefore, $x^{*}$ is unique.

Following the same arguments as in (14)-(18), one can show that

$$
\begin{aligned}
\left\|x_{2 n+1}-x^{*}\right\|^{2}= & \left\|P_{C_{2 n}}\left(w_{2 n}-\tau_{2 n} \nabla f_{2 n}\left(\bar{x}_{2 n}\right)\right)-x^{*}\right\|^{2} \\
\leq & \left\|w_{2 n}-x^{*}\right\|^{2}-2 \tau_{2 n}\left\langle\nabla f_{2 n}\left(\bar{x}_{2 n}\right), \bar{x}_{2 n}-x^{*}\right\rangle-\left\|x_{2 n+1}-\bar{x}_{2 n}\right\|^{2} \\
& -\left\|\bar{x}_{2 n}-w_{2 n}\right\|^{2}-2\left\langle\bar{x}_{2 n}-w_{2 n}\right. \\
& \left.+\tau_{2 n} \nabla f_{2 n}\left(\bar{x}_{2 n}\right), x_{2 n+1}-\bar{x}_{2 n}\right\rangle \\
\leq & \left\|w_{2 n}-x^{*}\right\|^{2}-\frac{2 \mu l}{\|A\|^{2}}\left\|\left(I-P_{Q_{2 n}}\right) A \bar{x}_{2 n}\right\|^{2} \\
& -\left(1-\mu^{2}\right)\left\|w_{2 n}-\bar{x}_{2 n}\right\|^{2} \\
\leq & \left\|w_{2 n}-x^{*}\right\|^{2} .
\end{aligned}
$$

Therefore,

$$
\left\|x_{2 n+1}-x^{*}\right\| \leq\left\|w_{2 n}-x^{*}\right\|=\left\|x_{2 n}-x^{*}\right\|
$$

Thus,

$$
\lim _{n \rightarrow \infty}\left\|x_{2 n+1}-x^{*}\right\|=0
$$

Therefore, $\lim _{n \rightarrow \infty} x_{n}=x^{*}$ and the desired result is obtained.

We give the following remark on our results.

Remark 3.4 (a) When vanilla inertial extrapolation step (the case when $w_{n}$ in (11) is computed as $\left.w_{n}=x_{n}+\theta_{n}\left(x_{n}-x_{n-1}\right), \forall n \geq 1\right)$ is added to methods for solving SCFP (1), the Fejér monotonicity of the generated sequence $\left\{x_{n}\right\}$ with respect to $S$ is lost. Here in our results in Lemma 3.2, we recover the Fejér monotonicity of $\left\{x_{2 n}\right\}$ with respect to $S$. This is one of the interesting properties of methods with alternated extrapolation step for solving SCFP (1).

(b) Our methods of proof in Lemma 3.2 and Theorem 3.3 are simpler and different from the methods of proof given in other papers (see, e.g., [16-18,44-47,51]) which solve SCFP (1) using methods with vanilla inertial extrapolation step.

\section{Numerical experiments}

In this section, we use the SCFP (1) to model two real problems, the first is the recovery of a sparse signal and the second is image deblurring. 
We make use of the well-known LASSO problem [48] which is the following.

$$
\min \left\{\frac{1}{2}\|A x-b\|_{2}^{2}: x \in \mathbb{R}^{k},\|x\|_{1} \leq t\right\},
$$

where $A \in \mathbb{R}^{m \times k}, m<k, b \in \mathbb{R}^{m}$ and $t>0$. This problem, (33) exhibits the potential of finding a sparse solution of the SCFP (1) due to the $\ell_{1}$ constraint.

Example 4.1 The first problem is focused in finding a sparse solution of the SCFP (1). We illustrate the advantages of our proposed scheme by comparing it with some related results in the literature, such as the methods in [23,40,50]. For the experiments the matrix $A$ is generated from a normal distribution with mean zero and one variance. The true sparse signal $x^{*}$ is generated from uniformly distribution in the interval $[-2,2]$ with random $K$ position nonzero while the rest is kept zero. The sample data $b=A x^{*}$ (no noise is assumed).

In the algorithm's implementations we choose the following parameters $\gamma=1$, $l=\mu=0.5$ and the constant stepsize $0.9 *(2 / L)$ for the relaxed CQ algorithm [50]. This parameters choices are arbitrary and valid theoretically, and here the goal is just to illustrate the performance of the methods. Clearly in a real-world scenario, one should have a deep investigation which involves intensive numerical simulations that can guaranteed optimal and performances. We limit the iterations number to 1000 and report the "Err" which is defined as $\left\|x_{n+1}-x_{n}\right\|$. We also report the the objective function value ("O $\mathrm{bj}$ ").

Under certain condition on matrix $A$, the solution of the minimization problem (33) is equivalent to the $\ell_{0}$-norm solution of the underdetermined linear system. For the considered SCFP (1), we define $C=\left\{x \in \mathbb{R}^{k}:\|x\|_{1} \leq t\right\}$ and $Q=\{b\}$. Instead of projecting onto the closed and convex set $C$ (there exists no closed formula), we use subgradient projection. So, define the convex function $c(x):=\|x\|_{1}-t$ and let $C_{n}$ be

Table 1 Numerical results obtained by all 4 CQ variants with $m=120, n=512$

\begin{tabular}{lllll}
\hline$K$-sparse signal & Methods & CPU time & Obj. & Err. \\
\hline$K=10$ & Alg. [23] & 0.422092 & 0.0011 & 0.0677 \\
& [40, Alg. 4.1.] & 0.411590 & 0.0011 & 0.0677 \\
Alg. [50] & 0.149126 & 0.0014 & 0.1066 \\
Alg. 1 & 0.021317 & 0.000765 & 0.00595 \\
Alg. [23] & [40, Alg. 4.1.] & 0.432794 & 0.0045 & 0.1205 \\
$K=30$ & Alg. [50] & 0.130378 & 0.0045 & 0.1205 \\
& Alg. 1 & 0.0319960 & 0.0083 & 0.2396 \\
& Alg. [23] & 0.402821 & 0.00047 & 0.01128 \\
& [40, Alg. 4.1.] & 0.406450 & 0.0091 & 0.1261 \\
& Alg. [50] & 0.141216 & 0.0176 & 0.1261 \\
& Alg. 1 & 0.0235084 & 0.0108 & 0.3529 \\
\hline
\end{tabular}


defined by

$$
C_{n}=\left\{x \in \mathbb{R}^{k}: c\left(w_{n}\right)+\left\langle\xi_{n}, x-w_{n}\right\rangle \leq 0\right\}
$$

where $\xi_{n} \in \partial c\left(w_{n}\right)$. It can be easily seen that the subdifferential $\partial c$ at $x \in \mathbb{R}^{k}$ is (defined element wise)

$$
[\partial c(x)]_{i}= \begin{cases}1, & x_{i}>0 \\ -1 \text { or } 1, & x_{i}=0 \\ -1, & x_{i}\end{cases}
$$

Now, the orthogonal projection of a point $x \in \mathbb{R}^{k}$ onto $C_{n}$ can be calculated by the following,

$$
P_{C_{n}}(x)= \begin{cases}x, & c\left(w_{n}\right)+\left\langle\xi_{n}, x-w_{n}\right\rangle \leq 0, \\ x-\frac{c\left(w_{n}\right)+\left\langle\xi_{n}, x-w_{n}\right\rangle}{\left\|\xi_{n}\right\|^{2}} \xi_{n}, & \text { otherwise. }\end{cases}
$$
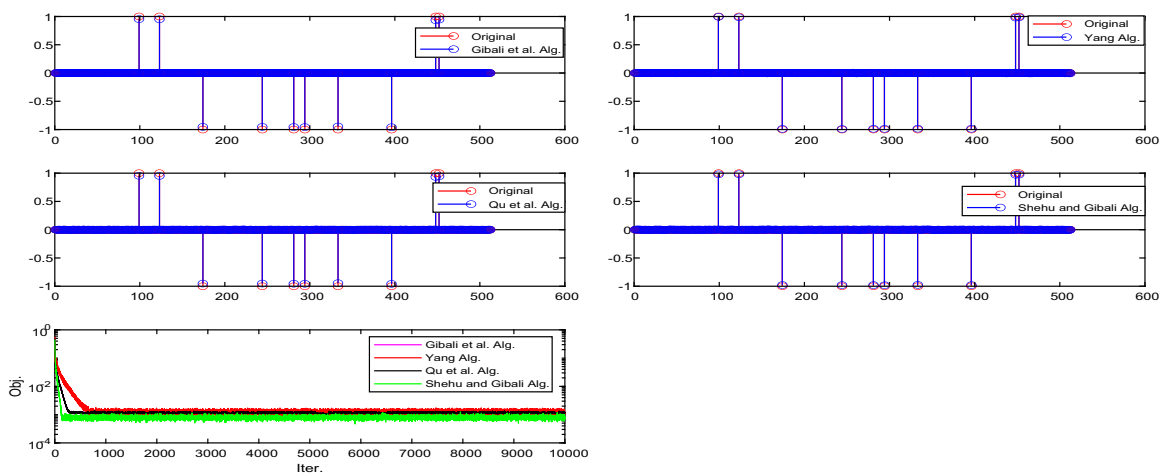

Fig. 1 The recovered sparse signal versus the original for the 4 CQ variants with $m=120, n=512$ and $K=10$
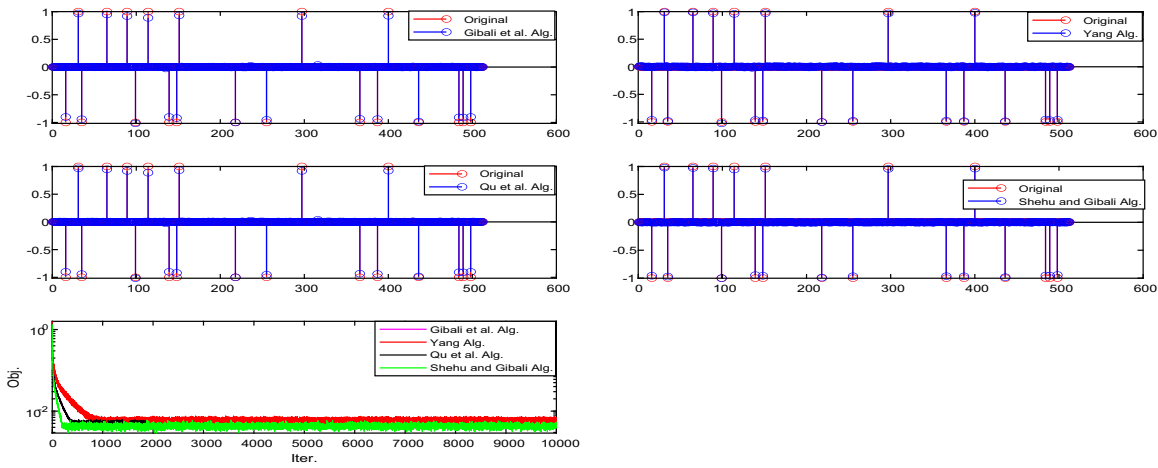

Fig. 2 The recovered sparse signal versus the original for the $4 \mathrm{CQ}$ variants with $m=120, n=512$ and $K=20$ 

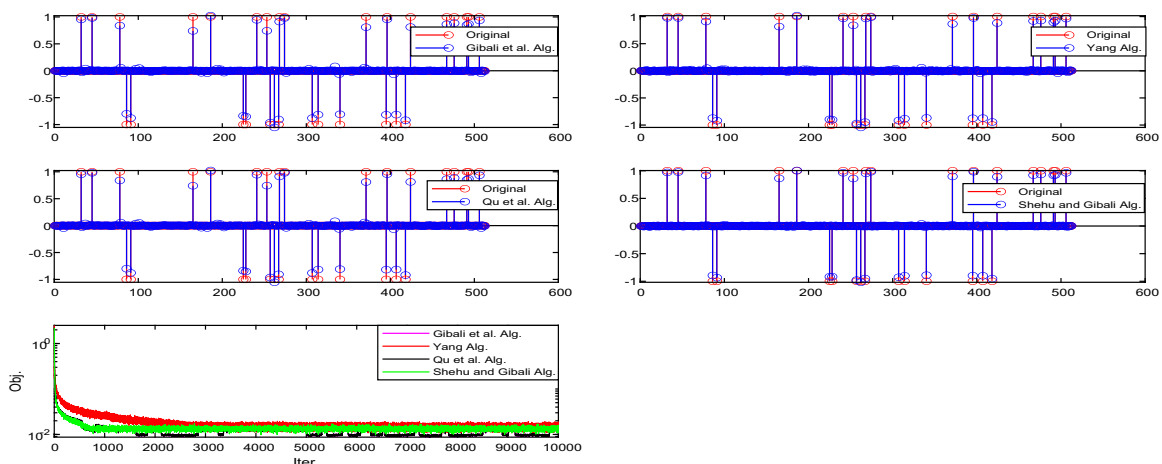

Fig. 3 The recovered sparse signal versus the original for the 4 CQ variants with $m=120, n=512$ and $K=30$

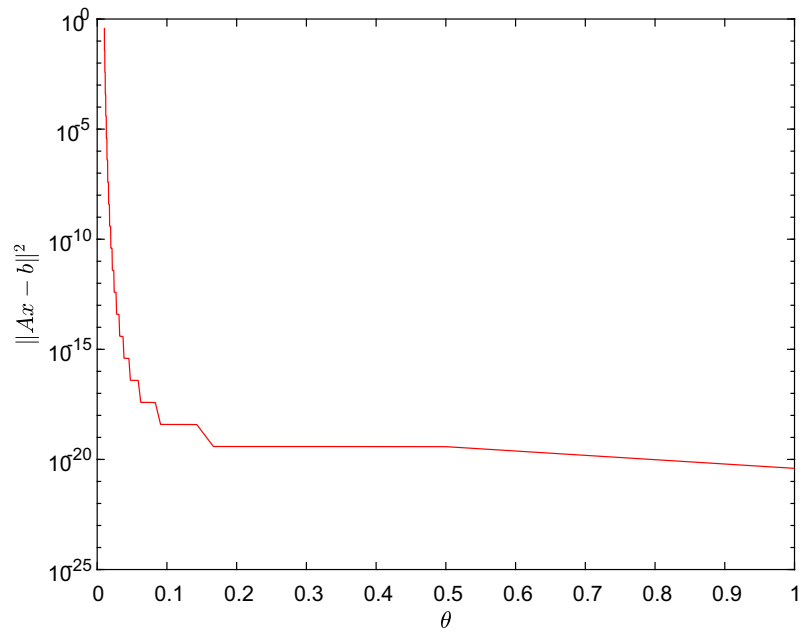

Fig. 4 The objective function value for different values of $\left\{\theta_{n}\right\}_{n=1}^{\infty}$

In Table 1 we summarize the results and in Figs. 1, 2 and 3 we plot the exact $K$ sparse signal against the recovered signals and the objective function values obtained by the different methods. One can clearly see that the inertial term plays as a significant role in achieving a better solution with respect to a lower objective value and CPU time for the same number of iterations.

Next for $K=20$ we illustrate the influence of the inertial parameter $\theta$ as it approaches 1 as a function of $\mu \rightarrow 0$ (taken as $\frac{1}{n}$ ). In Fig. 4 we plot the value of the objective function $\frac{1}{2}\|A x-b\|_{2}^{2}$ after 1000 iterations for any value of $\left\{\theta_{n}\right\}_{n=1}^{\infty}$ and other parameters are chosen as above.

Example 4.2 In this example we wish to apply our algorithm to image deblurring problem. Given a convolution matrix $A \in \mathbb{R}^{m \times k}$ and an unknown original image $x \in \mathbb{R}^{k}$, we get $b \in \mathbb{R}^{m}$, which is the known degraded observation. We also include unknown 


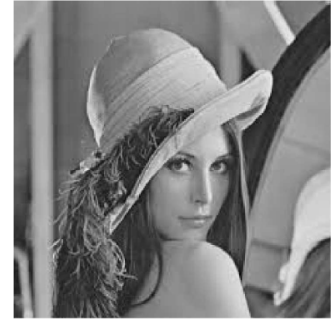

(a) Original

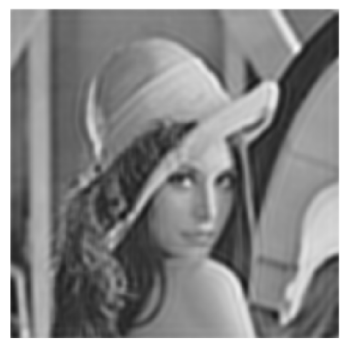

(c) $[40$, Alg. 4.1.]

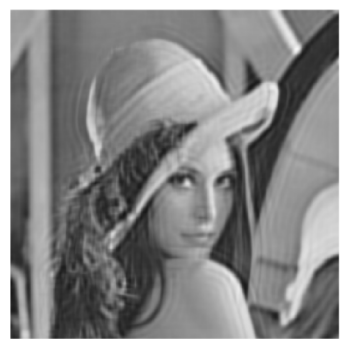

(f) $[35, \operatorname{Alg} .1]$

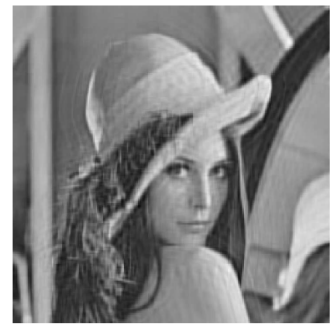

(i) Alg. 1

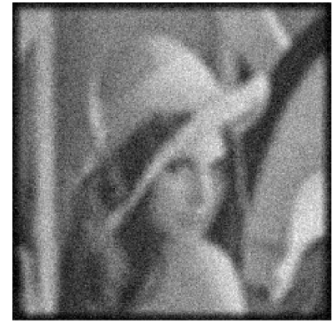

(b) Blurred and noisy image

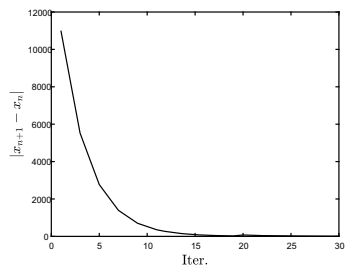

(d) $\left\|x_{n+1}-x_{n}\right\|$

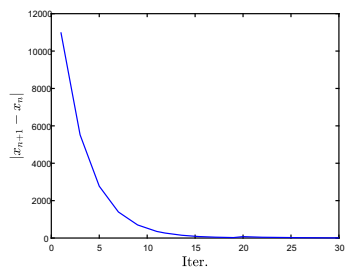

(g) $\left\|w_{n}-x_{n}\right\|$

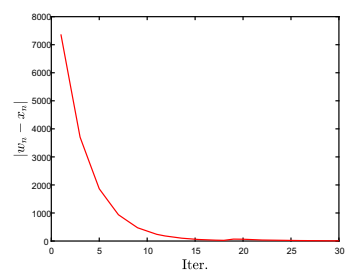

(j) $\left\|w_{n}-x_{n}\right\|$ (e) SNR
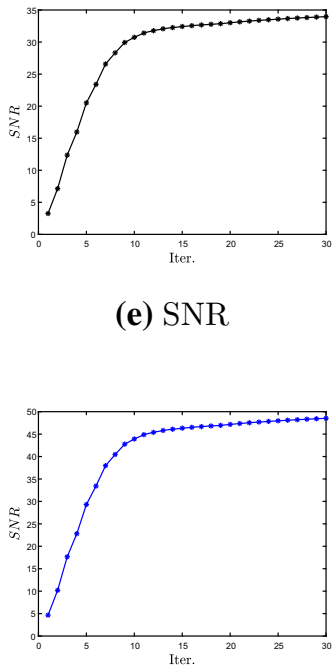

(h) SNR

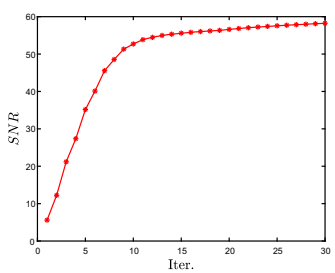

(k) SNR

Fig. 5 Recovered images via the different algorithms 
Table 2 Execution time of the different algorithms

\begin{tabular}{ll}
\hline Algorithms & CPU time \\
\hline [35, Alg. 1] & 28.435 \\
[40, Alg. 4.1.] & 36.445 \\
Alg. 1 & 24.2813 \\
\hline
\end{tabular}

additive random noise $v \in \mathbb{R}^{m}$ and get the following image recovery problem.

$$
A x=b+v .
$$

This problem can clearly fits into the setting of SCFP with $C=\mathbb{R}^{k}$, if no noise is included in the observed image $b$ then $Q=\{b\}$ is a singleton and otherwise $Q=$ $\left\{y \in \mathbb{R}^{m} \mid\|y-(b+v)\| \leq \varepsilon\right\}$ for small enough $\varepsilon>0$.

We illustrate the effectiveness and performance of our proposed Algorithm 1 compared with [40, Alg. 4.1.] and the very recent result of Padcharoen et al. [35, Alg. 1] which is the inertial Tseng method. The test image is the Lenna image (https://en.wikipedia. org/wiki/Lenna) which went through a $9 \times 9$ Gaussian random blur and random noise. Clearly this problem's structure differs from Example 4.1 but for simplicity we choose for Algorithm 1 compared with [40, Alg. 4.1.] the same parameters settings and for [35, Alg. 1] we choose the same choices as the authors did, that is, the inertial term $\alpha_{n}=0.9$ and the step size $\lambda_{n}=0.5-\frac{150 n}{1000 n+100}$. In Figs. $5(\mathrm{a})-(\mathrm{k})$ we report all results that include the recovered images via the different algorithms, the difference between successive iterations and the signal to noise ratio $\left(\mathrm{SNR}=10 \log \frac{\|x\|_{2}^{2}}{\left\|x-x_{n}\right\|_{2}^{2}}\right)$ with respect to the number of iterations.

The CPU time in seconds of the tested algorithms is reported in Table 2.

From Figs. 5 and Table 2 it can be seen that the inertial methods: Algorithm 1 and [35, Alg. 1] generate reasonable and compatible results after only 30 iterations compared with the non-inertial method [40, Alg. 4.1.]. The two major advantages of our proposed Algorithm 1 compared with the other two algorithms is the higher SNR value and lower CPU time for generating the recovered image.

\section{Final remarks}

In this paper, we give global convergence result for Split Convex Feasibility problem using relaxed CQ method with alternated inertial extrapolation step. Our result extend and generalize some existing results in the literature and the primary numerical results indicate that our proposed method outperforms most existing relaxed CQ method for solving SCFP.

Acknowledgements Open access funding provided by Institute of Science and Technology (IST Austria). The authors are grateful to the referees for their insightful comments which have improved the earlier version of the manuscript greatly. The first author has received funding from the European Research Council (ERC) under the European Union's Seventh Framework Program (FP7 - 2007-2013) (Grant agreement No. 616160). 
Open Access This article is licensed under a Creative Commons Attribution 4.0 International License, which permits use, sharing, adaptation, distribution and reproduction in any medium or format, as long as you give appropriate credit to the original author(s) and the source, provide a link to the Creative Commons licence, and indicate if changes were made. The images or other third party material in this article are included in the article's Creative Commons licence, unless indicated otherwise in a credit line to the material. If material is not included in the article's Creative Commons licence and your intended use is not permitted by statutory regulation or exceeds the permitted use, you will need to obtain permission directly from the copyright holder. To view a copy of this licence, visit http://creativecommons.org/licenses/by/4.0/.

\section{References}

1. Abass, H. A., Aremu, K. O., Jolaoso, L. O., Mewomo, O. T.: An inertial forward-backward splitting method for approximating solutions of certain optimization problems. J. Nonlinear Funct. Anal. Article ID 6 (2020)

2. Alvarez, F., Attouch, H.: An inertial proximal method for maximal monotone operators via discretization of a nonlinear oscillator with damping. Set-Valued Anal. 9, 3-11 (2001)

3. Bauschke, H.H., Borwein, J.M.: On projection algorithms for solving convex feasibility problems. SIAM Rev. 38, 367-426 (1996)

4. Bauschke, H. H., Combettes, P. L.: Convex analysis and monotone operator theory in Hilbert spaces, CMS Books in Mathematics/Ouvrages de Mathématiques de la SMC. Springer, New York, (2011). xvi+468 pp. ISBN: 978-1-4419-9466-0

5. Beck, A., Teboulle, M.: A fast iterative shrinkage-thresholding algorithm for linear inverse problems. SIAM J. Imaging Sci. 2, 183-202 (2009)

6. Bot, R.I., Csetnek, E.R., Hendrich, C.: Inertial Douglas-Rachford splitting for monotone inclusion. Appl. Math. Comput. 256, 472-487 (2015)

7. Bot, R.I., Csetnek, E.R.: An inertial alternating direction method of multipliers. Minimax Theory Appl. 1, 29-49 (2016)

8. Bot, R.I., Csetnek, E.R.: An inertial forward-backward-forward primal-dual splitting algorithm for solving monotone inclusion problems. Numer. Alg. 71, 519-540 (2016)

9. Byrne, C.: Iterative oblique projection onto convex sets and the split feasibility problem. Inverse Problems 18, 441-453 (2002)

10. Byrne, C.: A unified treatment of some iterative algorithms in signal processing and image reconstruction. Inverse Problems 20, 103-120 (2004)

11. Censor, Y., Bortfeld, T., Martin, B., Trofimov, A.: A unified approach for inversion problems in intensity-modulated radiation therapy. Phys. Med. Biol. 51, 2353-2365 (2006)

12. Censor, Y., Elfving, T.: A multiprojection algorithm using Bregman projections in a product space. Numer. Alg. 8, 221-239 (1994)

13. Censor, Y., Elfving, T., Kopf, N., Bortfeld, T.: The multiple-sets split feasibility problem and its applications for inverse problems. Inverse Problems 21, 2071-2084 (2005)

14. Censor, Y., Lent, A.: An iterative row-action method for interval convex programming. J. Optim. Theory Appl. 34, 321-353 (1981)

15. Censor, Y., Motova, A., Segal, A.: Perturbed projections and subgradient projections for the multiplesets split feasibility problem. J. Math. Anal. Appl. 327, 1244-1256 (2007)

16. Chuang, C.-S.: Hybrid inertial proximal algorithm for the split variational inclusion problem in Hilbert spaces with applications. Optimization 66(5), 777-792 (2017)

17. Dang, Y., Gao, Y.: The strong convergence of a KM-CQ-like algorithm for a split feasibility problem. Inverse Problems 27, 9 (2011)

18. Dang, Y.-Z., Sun, J., Xu, H.K.: Inertial accelerated algorithms for solving a split feasibility problem. J. Ind. Manag. Optim. 13, 1383-1394 (2017)

19. Dang, Y.-Z., Sun, J., Zhang, S.: Double projection algorithms for solving the split feasibility problems. J. Ind. Manag. Optim. 15, 2023-2034 (2019)

20. Dong, Q.L., Tang, Y.C., Cho, Y.J., Rassias, ThM: “Optimal” choice of the step length of the projection and contraction methods for solving the split feasibility problem. J. Global Optim. 71, 341-360 (2018)

21. Dong, Q.L., Yuan, H.B., Cho, Y.J., Rassias, ThM: Modified inertial Mann algorithm and inertial CQalgorithm for nonexpansive mappings. Optim. Lett. 12, 87-102 (2018) 
22. Fukushima, M.: A relaxed projection method for variational inequalities. Math. Program. 35, 58-70 (1986)

23. Gibali, A., Liu, L.-W., Tang, Y.-C.: Note on the modified relaxation CQ algorithm for the split feasibility problem. Optim. Lett. 12, 817-830 (2018)

24. Gibali, A., Ha, N.H., Thuong, N.T., Trang, T.H., Vinh, N.T.: Polyaks gradient method for solving the split convex feasibility problem and its applications. J. Appl. Numer. Optim. 1, 145-156 (2019)

25. Goebel, K., Kirk, W.A.: On Metric Fixed Point Theory. Cambridge University Press, Cambridge (1990)

26. Hendrickx, J.M., Olshevsky, A.: Matrix $p$-norms are NP-hard to approximate if $P \neq 1,2, \infty$. SIAM J. Matrix Anal. Appl. 31, 2802-2812 (2010)

27. Iutzeler, F., Hendrickx, J.M.: A generic online acceleration scheme for optimization algorithms via relaxation and inertia. Optim. Methods Softw. 34, 383-405 (2019)

28. Iutzeler, F., Malick, J.: On the proximal gradient algorithm with alternated inertia. J. Optim. Theory Appl. 176, 688-710 (2018)

29. Jouymandi, Z., Moradlou, F.: Extragradient and linesearch methods for solving split feasibility problems in Hilbert spaces. Math. Methods Appl. Sci. 42, 4343-4359 (2019)

30. López, G., Martín-Márquez, V., Wang, F., Xu, H.-K.: Solving the split feasibility problem without prior knowledge of matrix norms. Inverse Problems 28, 18 (2012)

31. Lorenz, D.A., Pock, T.: An inertial forward-backward algorithm for monotone inclusions. J. Math. Imaging Vision 51, 311-325 (2015)

32. Maingé, P.E.: Inertial iterative process for fixed points of certain quasi-nonexpansive mappings. Set Valued Anal. 15, 67-79 (2007)

33. Malitsky, Y., Pock, T.: A first-order primal-dual algorithm with linesearch. SIAM J. Optim. 28, 411-432 (2018)

34. Moudafi, A., Oliny, M.: Convergence of a splitting inertial proximal method for monotone operators. J. Comput. Appl. Math. 155, 447-454 (2003)

35. Padcharoen, A., Kitkuan, D., Kumam, W., Kumam, P.: Tseng methods with inertial for solving inclusion problems and application to image deblurring and image recovery problems. Comput. Math. Methods (2020). https://doi.org/10.1002/cmm4.1088

36. Mu, Z., Peng, Y.: A note on the inertial proximal point method. Stat. Optim. Inf. Comput. 3, 241-248 (2015)

37. Nesterov, Y.: A method for solving the convex programming problem with convergence rate $O\left(1 / k^{2}\right)$. Dokl. Akad. Nauk SSSR 269, 543-547 (1983)

38. Ogbuisi, F.U., Shehu, Y.: A projected subgradient-proximal method for split equality equilibrium problems of pseudomonotone bifunctions in Banach spaces. J. Nonlinear Var. Anal. 3, 205-224 (2019)

39. Polyak, B.T.: Some methods of speeding up the convergence of iteration methods. U.S.S.R. Comput. Math. Math. Phys. 4, 1-17 (1964)

40. Qu, B., Xiu, N.: A note on the CQ algorithm for the split feasibility problem. Inverse Problems 21, 1655-1665 (2005)

41. Schöpfer, F., Schuster, T., Louis, A.K.: An iterative regularization method for the solution of the split feasibility problem in Banach spaces. Inverse Problems 24, 055008 (2008)

42. Shehu, Y.: Iterative methods for split feasibility problems in certain Banach spaces. J. Nonlinear Convex Anal. 16, 2351-2364 (2015)

43. Shehu, Y., Iyiola, O.S., Enyi, C.D.: An iterative algorithm for solving split feasibility problems and fixed point problems in Banach spaces. Numer. Algorithm 72, 835-864 (2016)

44. Shehu, Y., Vuong, P.T., Cholamjiak, P.: A self-adaptive projection method with an inertial technique for split feasibility problems in Banach spaces with applications to image restoration problems. J. Fixed Point Theory Appl. 21(2), 24 (2019)

45. Shehu, Y., Iyiola, O.S.: Convergence analysis for the proximal split feasibility problem using an inertial extrapolation term method. J. Fixed Point Theory Appl. 19, 2483-2510 (2017)

46. Suantai, S., Pholasa, N., Cholamjiak, P.: Relaxed CQ algorithms involving the inertial technique for multiple-sets split feasibility problems. Rev. R. Acad. Cienc. Exactas Fís. Nat. Ser. A Mat. RACSAM 113, 1081-1099 (2019)

47. Suantai, S., Pholasa, N., Cholamjiak, P.: The modified inertial relaxed CQ algorithm for solving the split feasibility problems. J. Ind. Manag. Optim. 14, 1595-1615 (2018)

48. Tibshirani, R.: Regression shrinkage and selection via the LASSO. J. R. Stat. Soc. Ser. B Stat. Methodol. 58, 267-288 (1996) 
49. $\mathrm{Xu}, \mathrm{H} . \mathrm{K}$.: Iterative methods for the split feasibility problem in infinite-dimensional Hilbert spaces. Inverse Problems 26, 105018 (2010)

50. Yang, Q.: The relaxed CQ algorithm solving the split feasibility problem. Inverse Problems 20, 12611266 (2004)

51. Yao, Y., Qin, X., Yao, J.-C.: Convergence analysis of an inertial iterate for the proximal split feasibility problem. J. Nonlinear Convex Anal. 20, 489-498 (2019)

52. Yu, H., Zhan, W., Wang, F.: The ball-relaxed CQ algorithms for the split feasibility problem. Optimization 67, 1687-1699 (2018)

Publisher's Note Springer Nature remains neutral with regard to jurisdictional claims in published maps and institutional affiliations. 